\title{
Detect and Analyze Face Parts Information using Viola- Jones and Geometric Approaches
}

\author{
Amr El Maghraby \\ Computers \& \\ Systems Eng \\ Zagazig University
}

\author{
Mahmoud Abdalla \\ Communication Eng \\ Zagazig University
}

\author{
Othman Enany \\ Computers \& \\ Systems Eng \\ Zagazig University
}

\author{
Mohamed Y. El \\ Nahas \\ Computers \& \\ Systems Eng \\ Elazhar University
}

\begin{abstract}
This paper presents a new face parts information analyzer, as a promising model for detecting faces and locating the facial features in images. The main objective is to build fully automated human facial measurements systems from images with complex backgrounds. Detection of facial features such as eye, nose, and mouth is an important step for many subsequent facial image analysis tasks. The study covers the tasks detection, landmark localization and measurement facial part that have traditionally been approached as separate problems with different techniques.

Different set of techniques have been introduced recently, for example; principal component analysis, geometric modeling, auto-correlation, deformable template, neural networks, color analysis, window classifiers, view-based Eigen space methods, and elastic graph models. The study present a novel and simple model approach based on a mixture of techniques and algorithms in a shared pool based on Viola-Jones object detection framework algorithm combined with geometric and symmetric information of the face parts from the image in a smart algorithm. The study is a continued part of previous work [1] the proposed model is modestly applied with hundreds of face images taken under different lighting conditions, a number of general assumptions used in this research field are identified.
\end{abstract}

After analyzing and testing the new algorithm with hundreds of faces, several promising directions for future research are concluded.

\section{General Terms}

Image processing, human face detection Algorithms

\section{Keywords}

Face detection, Video frames, Viola- Jones, Skin detection, Skin color classification. Face detection, gender detection recognition, machine learning.

\section{INTRODUCTION}

It's a true challenge to build an automated system which equals human ability to detect faces and estimates human body dimensions from an image or a video. The conceptual and intellectual challenges of such a problem, because faces are non-rigid and have a high degree of variability in size, shape, color and texture. Numerous techniques have been developed and designed typically to deal with single images .Detect human appearance in image or track humans in videos become a very important task for many applications such as: auto focus in cameras, visual surveillance, traffic safety monitoring and human computer interaction [2].
From both neurological and computational point of view symmetry plays an important role in facial part dimensions as it has been demonstrated that an exceptional dimension reduction can be made by taking into account facial symmetry [3].

The present method usually returns the image location of a rectangular bounding box containing a face. is focusing to isolate the human body in a relatively clean background from input images or videos containing people roughly localized and applying a list of human-based measuring units on the human bodies.

This proposed algorithm goes beyond the limits of all existing technologies as it obtains the unique functional features by enabling the proposed model to work with different skin color tone, applying it to low-quality images, detecting faces with eye glasses, determining the position of facial parts (e.g. eye pupils, nose, lips, etc.) and detect several faces on one image is typically designed to deal with single images

\section{THE VIOLA-JONES OBJECT DETECTION FRAMEWORK}

The Viola-Jones object detection frame work [4] is the first object detection framework to provide competitive object detection rates in real-time proposed in 2001 by Paul Viola and Michael Jones. It was motivated primarily by the problem of face detection, although it can be trained to detect a variety of object classes. This algorithm is implemented in Open CV as cvHaarDetectObjects().Viola Jones detector become famous due to its open source implementation in the OpenCV library. In order to find an object of an unknown size is usually adopted to work this field that possesses a high efficiency and accuracy to locate the face region in an image. The Viola - Jones method contains three techniques:

1. Integral image for feature extraction the Haar-like features is rectangular type that is obtained by integral image

2. Adaboost is a machine-learning method for face detection [5], The word "boosted" means that the classifiers at every stage of the cascade are complex themselves and they are built out of basic classifiers using one of four boosting techniques (weighted voting).The Adaboost algorithm is a learning process that is a weak classification and then uses the weight value to learn and construct as a strong classification.

3. Cascade classifier used to combine many features efficiently. The word "cascade" in the classifier name means that the resultant classifier consists of several 
simpler classifiers (stages) that are applied subsequently to a region of interest until at some stage the candidate is rejected or all the stages are passed. Finally, the model can obtain the non-face region and face region after cascading each of strong classifiers

\subsection{Viola- Jones Upper body detection}

Accurate upper body detection improves the robustness and reduces the challenging task of detecting upper bodies from unconstrained still images. The cascade object detector uses the Viola-Jones algorithm to detect people's upper body. The model detects the upper-body region, which is defined as the head and shoulders area. This model uses Haar features [6] to encode the details of the head and shoulder region. Because it uses more features around the head, this model is more robust against pose changes, e.g. head rotations/tilts To Detect Upper Body in an Image Using the Upper Body Classification Model.

1- Create a detector object and set properties.

2- Read input image and detect upper body.

3- Annotate detected upper bodies.

\subsection{Viola-Jones Face Detection Algorithm}

Early efforts in face detection have dated back as early as the beginning of the 1970s, where simple heuristic and anthropometric techniques [7] Face detection techniques can be categorized into two major groups that are feature based approaches and image based approaches. Image based approaches use linear subspace method, neural networks and statistical approaches for face detection. Feature based approaches can be subdivided into low level analysis, feature analysis and active shape model.

Face detection is controlled by special trained scanning window classifiers Viola-Jones Face Detection Algorithm is the first real-time face detection system. There are three ingredients working in concert to enable a fast and accurate detection: the integral image for feature computation, Adaboost for feature selection and cascade for efficient computational resource allocation. .

\subsection{Viola-Jones Eye Detection Algorithm}

Eyes are detected based on the hypothesis that they are darker than other part of the face, finding eye analogue segments searching small patches in the input image that are roughly as large as an eye and are darker than their neighborhoods. a pair of potential eye regions is considered as eyes if it satisfies some constraints based on anthropological characteristics of human eyes [8] .To discard regions corresponding to eyebrows, the model uses the fact that the center part of an eye region is darker than other parts. Then a simple histogram analysis of the region is done for selecting eye regions since an eye region should exhibit two peaks while an eyebrow region shows only one. A final constraint is the alignment of the two major axis, so the two eye regions belong to the same line. The study propose a new algorithm for eyes' detection that uses Iris geometrical information for determining the whole image region containing an eye, and then applying the symmetry for selecting both eyes.

\subsubsection{Pupil detection in iris recognition system}

The iris has many properties which makes it the ideal biometric recognition component. The pupil is the dark circle at the center of your eye as shown in "figure1". It is surrounded by the iris, which is the colored part of eye. The pupil allows light to enter the eye. Light enters through the pupil, passes through the lens, and is focused onto the retina. A biometric system provides automatic identification of an individual based on a unique feature or characteristic possessed by the individual. Iris recognition is regarded as the most reliable and accurate biometric identification system available. Most commercial iris recognition systems use patented algorithms developed by Daugman [9] and these algorithms are able to produce perfect recognition rates.

However, published results have usually been produced under favourable conditions, and there have been no independent trials of the technology.

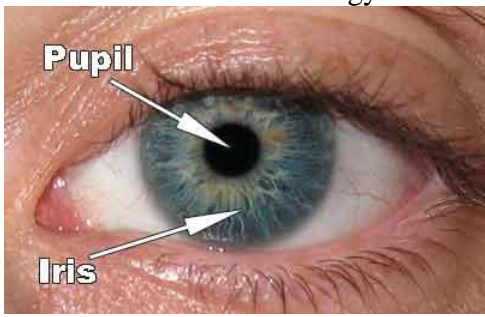

Figure 1: front view of human eye

the boundary of pupil is not quite circle and a small error in detecting this boundary will lead to lose some information surrounding the pupil. Another problem that makes most of the iris segmentation method fail to detect the pupil boundary is the head rotation or the eye rotation.[10]

\subsection{Viola-Jones nose Detection Algorithm}

Nose have three different local characteristics used as follows [11](i) Similarity of both sides: The left and right sides of nose are similar in a front-view face as shown in Fig. (1a), this property of similarity can be measured using Euclidean distance between both sides.(ii) Dark-White-Dark (DWD) property: the lower part of nose region is characterized by two dark nostrils and a light sub region due to the reflection of light on the nose as shown in "Figure 2".

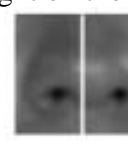

(a)

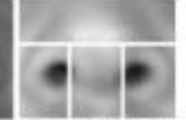

D W D

(b)

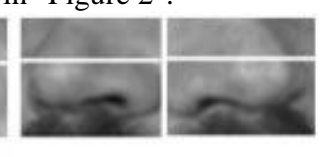

(c)
Figure 2: to the reflection of light on the nose

The two nostrils regions are less than the average of middle lighter sub region containing nose tip. (iii) The variation in lower/upper parts property: When the face is rotated some degrees for these two properties are despaired and the only clear property is the variation between lower part and upper part as shown in "Figure 2c". This variation can be measured by the variance in each part. Based on this analysis, a search is done for a certain region among the ten highest regions detected due to reflection of light at nose tip. Nose tip region is very bright as compared to other part of nose region. Due to the presence of very bright region, this region appears as black in binary image obtained in previous step because binary image and is generated by marking only dark pixels as white. To locate this region the algorithm find large connected black region in central region of localized nose image. Once this region is identified, lowest tip of this region is considered to be nose tip. On both sides of nose tips, same binary image 
is searched for nose edges and distance between these edges, is considered as nose width.

\subsection{Viola-Jones mouth Detection Algorithm}

Detection and Extraction features from the mouth region; this model is composed of weak classifiers, based on a decision stump, which uses Haar features to encode mouth details. Experimental results show that the algorithm is Face image division based on physical approximation of location of eyes, nose and mouth on face and can find out the mouth region rapidly. It is useful in a wide range; moreover, it is effectual for complex background such as public mouth detection.

\subsubsection{Lip Detection}

The basic concept of the proposed algorithm is to extract and then verify the desired components, including skins, lips, by applying some kind of color segmentation in addition to some geometry. Based on the color distribution of the lips and normal skins The lip detection method based on RGB chromaticity diagram is used to separate lip color from other colors (face skin color) by a simple effective color segmentation method [12] .Observing the pixels of lips found that the colors of lips range from dark red to purple under normal light condition. From the perspective of human visual perception, the lips are very easy to be differentiated from the face skins for any races of people because of their different contrasts in color. One of the advantages of these color spaces in skin detection is that they allow users to intuitively specify the boundary of the skin color class in terms of the hue and saturation. Similarly, RGB to $\mathrm{YCbCr}$ color space conversion is performed to find out threshold for skin region using the following equation. In order to detect skin color following set of rules have been found to be more accurate than other models $\mathrm{Y}=0.257 \mathrm{R}+0.504 \mathrm{G}+0.098 \mathrm{~B}+16$

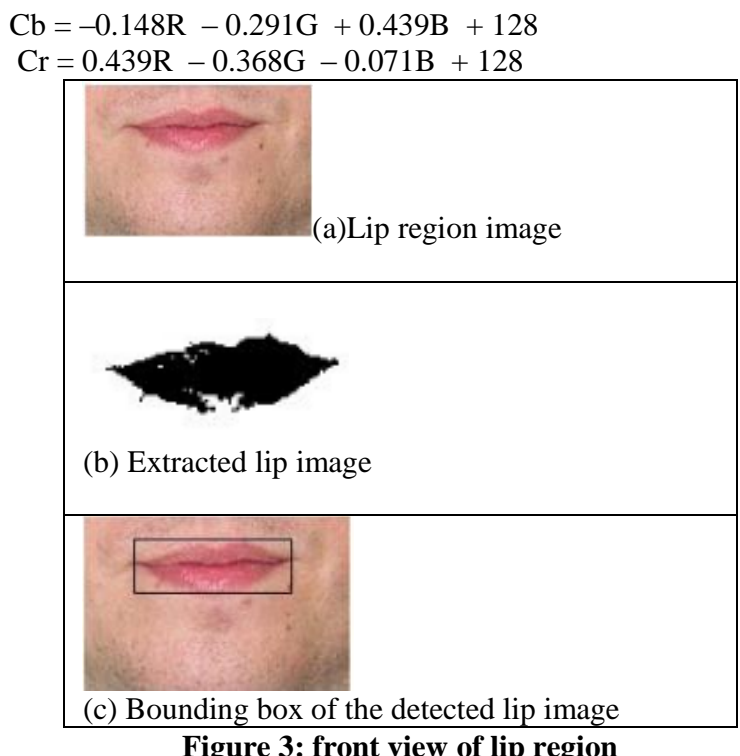

\section{Previous Work}

In Hybrid Face Detection System using Combination of Viola - Jones Method and Skin Detection [1] the cascade object detector framework proposed by Viola-Jones was extended. The main objective was to detect face parts in still images or videos, especially in complex background images, by using Viola Jones upper body model to detect near-frontal upperbodies as a region of interest. This is the primary detector where the high probability of finding the face instead of searching the entire image. In order to find an accurate face in that region of interest, Viola-Jones' face detector is used as a secondary detector to increase accuracy and reduces false negatives. Third detector pixel-based skin detection methods are applied on the region of interest which is not detecting a face using the secondary detector. The third detector classifies each pixel as skin or non-skin individually and independently from its neighbors and combines it with Viola - Jones upper body detection. This improves the performance of face detection systems in terms of increasing the face detection speed and decreasing false positive rate. The primary, secondary and third detectors are combined by this release and a single homogeneous set of face bounding-boxes are returned. The algorithm can detect a face in video as usual in each image as shown in "figure4".

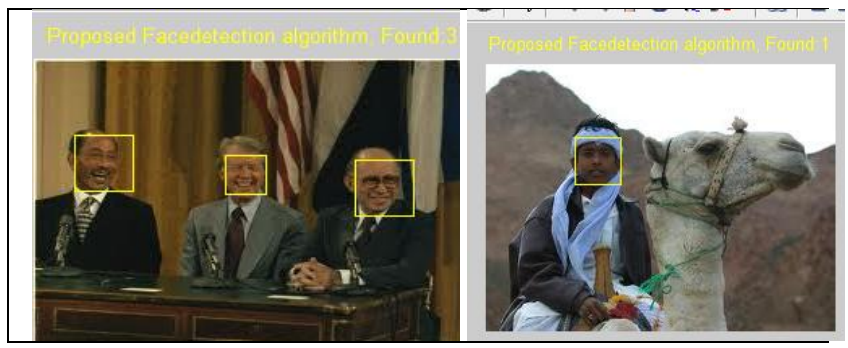

Figure 4: sample of our previous study Hybrid Face Detection System using Combination of Viola - Jones Method and Skin Detection

\section{PROPOSED FACE DETECTION FRAMEWORK}

The proposed method illustrated in this paper is applied as input after applying the detection algorithm using the previous work[1] which give a green box that surrounds the face as shown in "figure5", and this region will be the area of interest in the present algorithm .

The new model determines the locations and sizes of human faces in digital images by extracting region of interest and apply Viola Jones proper object detector in the area of interest in order to limit search area. These techniques radically reduce computation time.

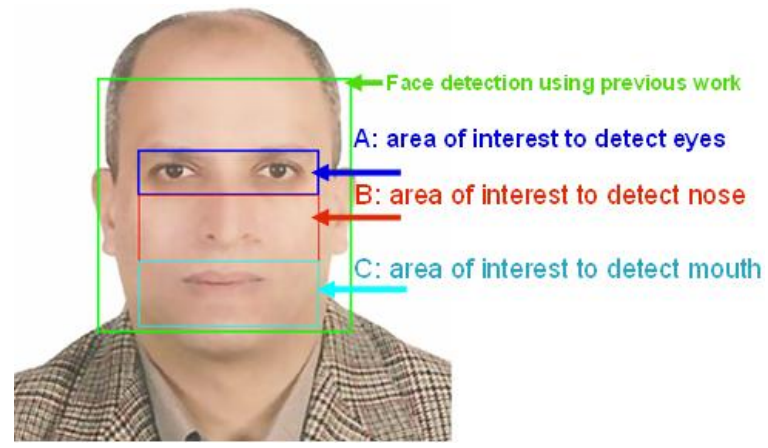

Figure 5: area of interest sub region detect face parts 


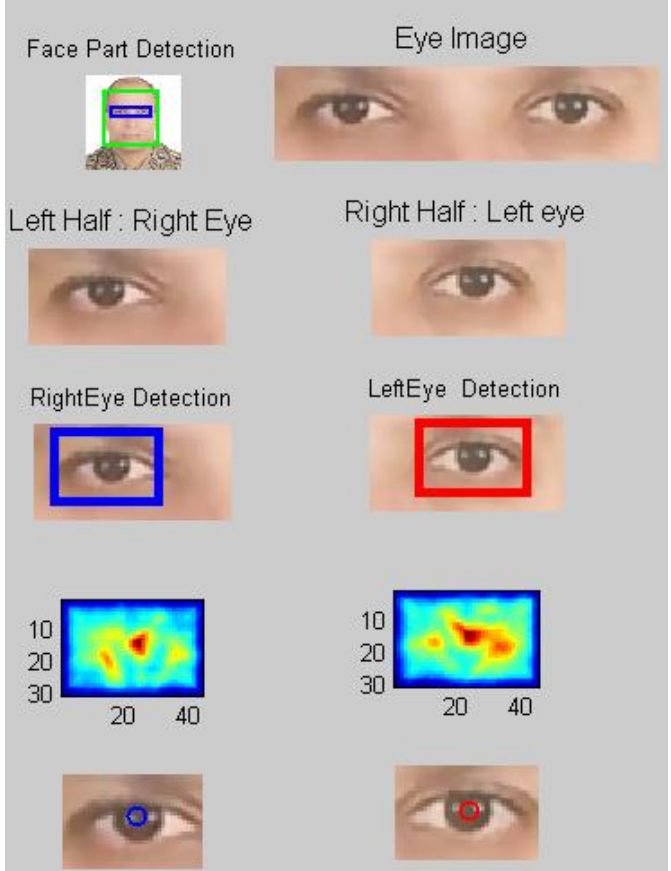

Figure 6: Eye detection Proposed algorithm Draw automatically eye pupil

As shown in "figure5" the face detection area are divided to three regions of interest calculated using the following; From the Detected Face, the model apply Viola - Jones Eye Pair Big to detect eye pair in the face detection area and create the region for performing the eyes search (A): area of interest to detect eye.

From the Detected area of eye pair the region for performing the nose search is created as 1.5 height of eye region and the same width of eye pair (B) area of interest to detect nose.

The region of the lower third of the face is isolated for performing the mouth search (c) area of interest to detect mouth.

To detect eye pupil the algorithm isolate the area of interest (A) in "figure 5" area of interest to detect eye by processing this region as shown in "figure 6" by dividing this region into two parts; left half contain right eye and a right half contain the left eye the study apply Viola - Jones right eye detector on right eye region to detect the right eye, and apply Viola - Jones left eye detector on left eye region to detect the left eye .Our last detection step is determining the location of eye pupil and determine the distance between them. Tthe study use the Iris recognition system which consists of an automatic segmentation system that is based on the Hough transform to be able to localize the circular iris pupil detection is based on the fact that pupil will have the highest black value relative to everything else in the iris region . From the iris region the study extract the maximum value corresponding to the pupil center. Furthermore, the distance between the center of pupils called pupillary distance (PD) use the following formula to calculate distance between two points .This will get center points of the two eyes as detected by the proposed algorithm and enable to calculate the distance between the two pupil axis using the following equation

pupillary distance $(\mathrm{PD})=\operatorname{sqrt}\left((\mathrm{y} 2-\mathrm{y} 1)^{\wedge} 2+(\mathrm{x} 2-\mathrm{x} 1)^{\wedge} 2\right)$;
Where $(\mathrm{x} 1, \mathrm{y} 1)$ and $(\mathrm{x} 2, \mathrm{y} 2)$ are coordinates of two pixels of the center of the iris of the eye as shown in "figure 7 ".

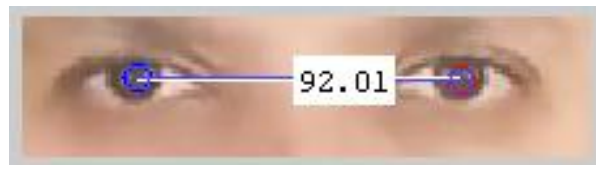

figure7: pupillary distance (PD)

Nose detection is highly important for face part detection in range images and is consider as simpler task than detecting a face because a nose is not as complex and variable as a face. In this paper, the proposed nose detection algorithm has the following three characteristics. First, it is based on The cascade object detector which uses the Viola-Jones algorithm to detect people's noses This model is composed of weak classifiers, based on a decision stump, which uses Haar features to encode nose details. Second, it can deal with both frontal and non-frontal poses. Finally, it is quite fast and accurate because the algorithm searching in a small region of the image (b)area of interest to detect the nose not the whole image .

To detect the nose within the face region. The nose provides a more accurate measure of the skin tone because it does not contain any background pixels. Viola-Jones algorithm is used as first step to Detect nose This model is composed of weak classifiers, based on a decision stump, which use Haar features to encode nose details The nose bounding box is relatively defined to the cropped face image. Adjusting the nose bounding box so that it is relative to the original image or video frame for detected supratip from the nose region. The model uses the following steps 1-the algorithm convert the nose detected color image from BGR to HSV . 2-Split the HSV color image into its separate Hue, Saturation and Value(Brightness). 3-Calculate maximum Brightness value return the pixel of supratip as show in "figure 8".

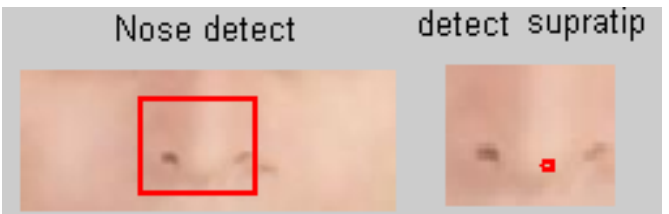

Figure8: Nose detect and supratip detect

For mouth detection This technique is a combination of existing Viola Jones mouth detection algorithm and physical location approximation is made in detected face to mouth. This method increased the accuracy of system and decreased consumed time as show in "figure 9"

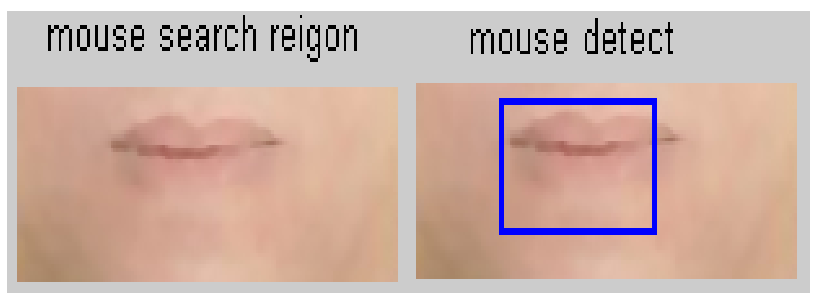

figure9: mouse search region and mouse detect 
The final result after applying geometric calculation for the distance between pupils and nose shown in "figure 10"

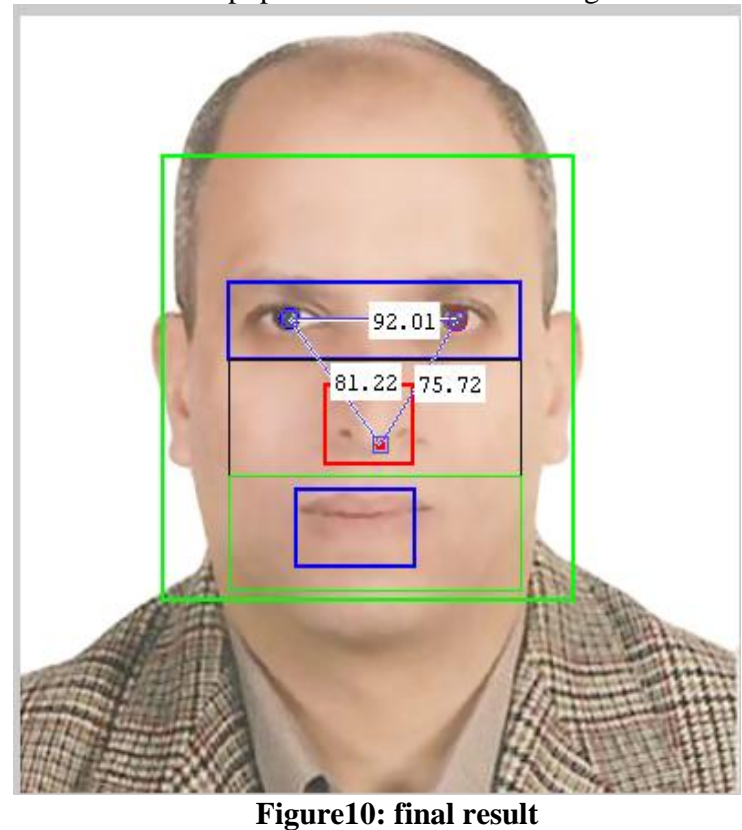

\section{EXPERIMENTAL RESULT}

In this section a detailed experimental comparison of the above stated approaches are being presented. Fifty test images were obtained in different lighting conditions and complex backgrounds with comparison of three algorithms on same images. Experimental results show that the algorithm is Face part division; based on physical approximation of location of eyes, nose and mouth on face and can find out the face part rapidly. It is also useful in a wide range. Moreover, it is effectual for complex background. The below figures are screen shots for the experimental comparison of the methods presented. The experimental test used 100 color images containing many faces with various complex backgrounds. The resulting detector automatically returned bounding-boxes fitting detecting faces parts appearing in images.

False positives (detecting the search object when there is none) false negatives (not detecting the object search that's present) as shown in "figure 11".

The "figure 12" demonstrated the statistical results of the experimental results on 100 test images representing faces at different imaging conditions. The detector applied the 2 different methods Viola Jones detection framework and Proposed algorithm .Experimental results showed that the proposed method improved face detection especially for people in different poses and difficult backgrounds as shown in "figure 13". The proposed algorithm clearly reduced false negative rates and detected face poses from the images at different imaging resolution and different lighting conditions.

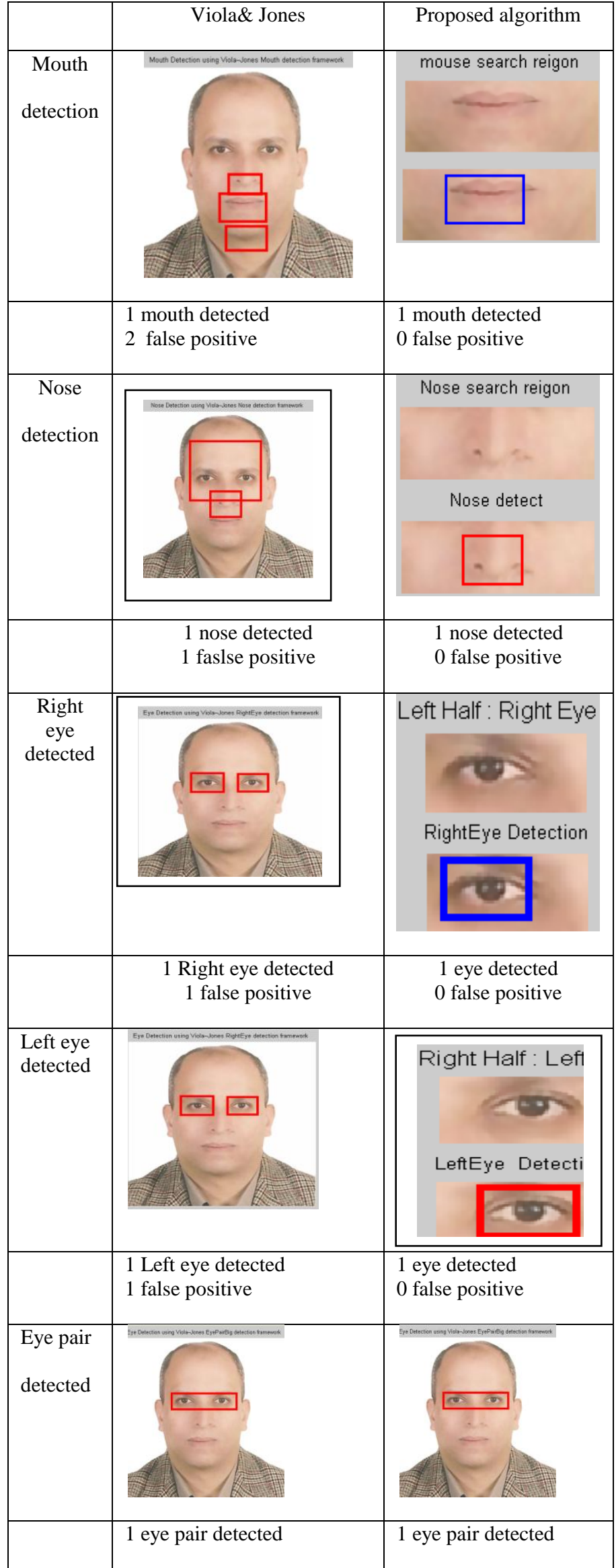

Figure11: Proposed algorithm results Draw automatically the returned bounding box around the face part detected 


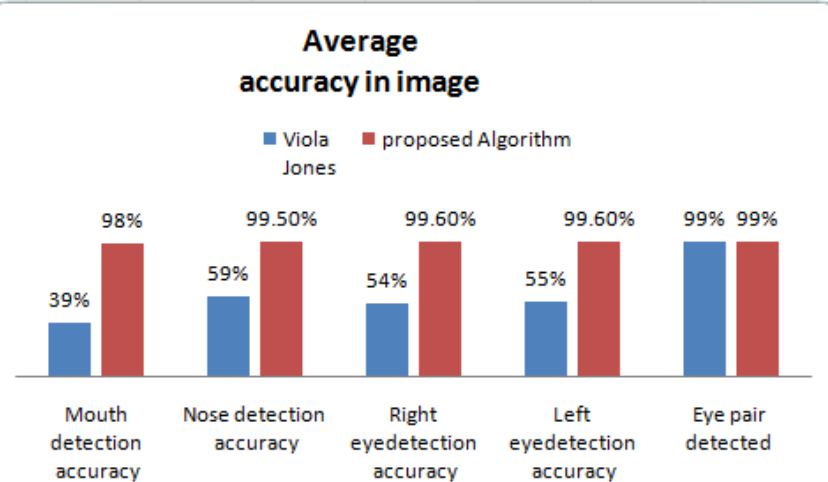

Figure 12: Comparison of Face part Detection Accuracy

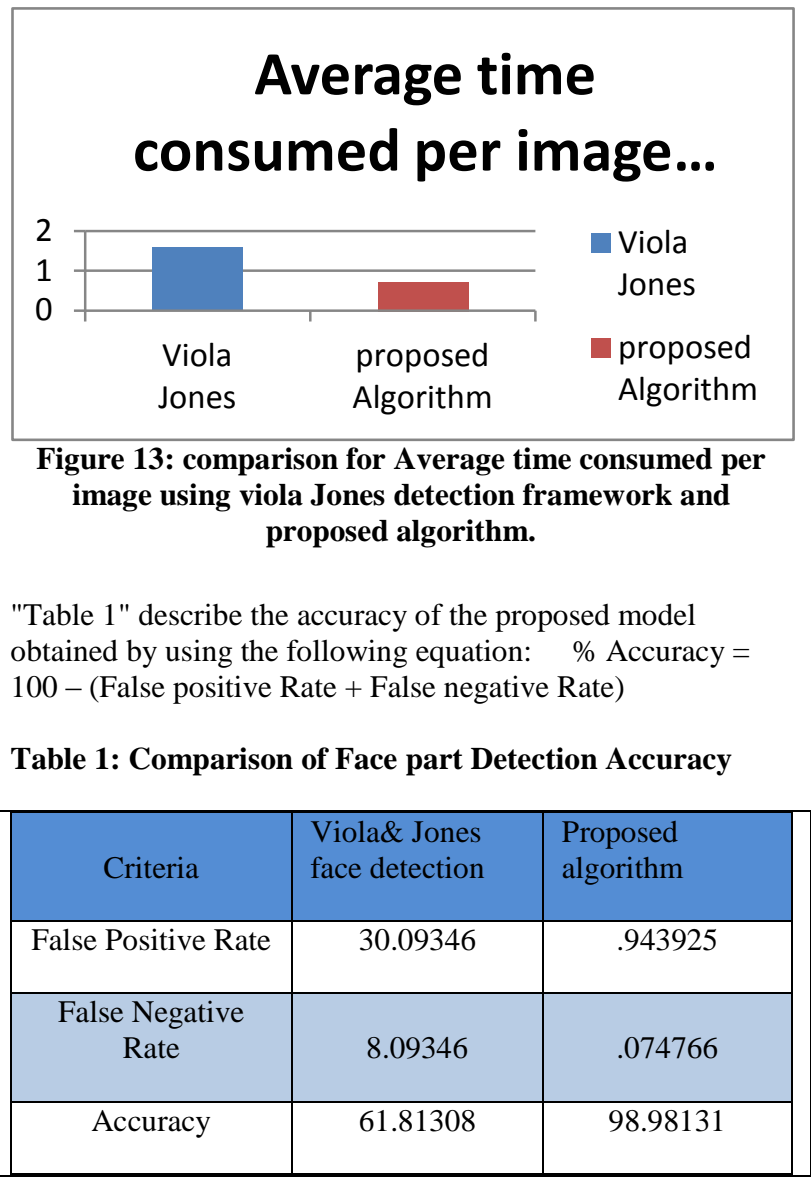

\section{CONCLUSION AND FUTURE SCOPE}

The study may be consider as a general application in computer vision and image processing, presented an efficient face part detection approach that achieves high detection accuracy with a low false positive rate.

In this paper, the first contribution is to build a novel face part detection model using combination of generic algorithms is presented to improve face part detection from images. The model is able to detect approximately 5 times faster than Viola approach while the detection rate is improved about $37.2 \%$.

The second contribution of this study, is to build fully automated accurate human facial measurements systems from images or video with complex backgrounds .
The experimental part in the study was implemented using Matlab environment, presenting a set of experiments on a difficult face detection dataset which has been widely studied. This research work was initiated as a part of research project for Human Actions Detection In Content-based Video Retrieval System. In the future this algorithm will be an essential part of a system which will identify human presence in video stream.

\section{ACKNOWLEDGMENTS}

Practical Application was done at Laboratory of Image Processing, Faculty of Engineering, Computers and Systems Engineering Dept., Zagazig University, , Egypt. Appreciation and gratitude to International Journal of Computer Applications Staff and research paper Referees.

\section{REFERENCES}

[1] A.Maghraby M.Abdalla O.Enany,Hybrid Face Detection System using Combination of Viola - Jones Method and Skin Detection, International Journal of Computer Applications (0975 - 8887) Volume 71- No.6, May 2013

[2] W. Zhao, R. Chellappa, A. Rosenfeld, and P. Phillips Face recognition:A literature survey. ACM Computing Surveys, pages 399-458, 2003.

[3] L. Sirovich and M. Meytlis. Symmetry, probability, and recognition inface space.PNAS - Proceedings of the National Academy of Sciences,106(17):6895-6899, April 2009.

[4] P.VIOLA and M.j.Jones, Robust real time face detection ,international journal of computer vision,57 (2004),

[5] K. T. Talele, S. Kadam, A. Tikare, Efficient Face Detection using Adaboost, "IJCA Proc on International Conference in Computational Intelligence", 2012

[6] Phillip I.W.,Dr. John F. FACIAL FEATURE DETECTION USING HAAR CLASSIFIERS,JCSC 21, (2006)

[7] T. Sakai, M. Nagao, and T. Kanade, Computer analysis and classification of photographs of human faces,in Proc. First USA_Japan Computer Conference, 1972, p. 2.7.

[8] Z.H. Zhou and X. Geng, "Projection functions for eye detection", Pattern Recognition 37, no 5, pp. 1049-1056, 2004

[9] Daugman J (2006) "Probing the uniqueness and randomness of IrisCodes: Results from 200 billion iris pair comparisons." Proceedings of the IEEE, 94(11),

[10] Gomai, G. A new approach for pupil detection in iris recognition system ,Computer Engineering and Technology (ICCET), 2010 (Volume:4)

[11] M. Hassaballah,KenjiMurakami, and Shun Ido, Eye and Nose Fields Detection From Gray Scale Facial Images, MVA2011 IAPR Conference on Machine Vision Applications, June 13-15, 2011, Nara, JAPAN

[12] S.Gurumurthy,B.K.Tripathy ,Design and Implementation of Face Recognition System in Matlab Using the Features of Lips,I.J. Intelligent Systems and Applications, 2012,8,30-36 